\title{
Haematoloechus combesi n. sp. (Trematoda, Haematoloechidae), parasite d'Amphibiens Anoures au Togo
}

\author{
par Gueorgui BATCHVAROV et Robert BOURGAT \\ Département de Biologie animale, Centre Universitaire, \\ avenue de Villeneuve, $F 66000$ Perpignan \\ et Ecole des Sciences, Université du Bénin, B.P. 1515, Lomé, Togo
}

Résumé.

H. combesi, parasite pulmonaire de Conraua derooi, se caractérise par la forme générale du corps, le rapport ventousaire $(2,7)$, l'ovaire et les testicules faiblement lobés, le fort développement vers l'avant des boucles utérines extracæcales et l'extension des vitellogènes dans la région post-testiculaire. L'ensemble de ces caractères permettent de distinguer $H$. combesi des autres espèces africaines du genre Haematoloechus, la plus voisine d'entre elles étant $H$. darcheni, parasite de Conraua crassipes au Gabon.

\section{Summary.}

Haematoloechus combesi n. sp. (Trematoda, Haematoloechidae), parasite of Anura Amphibians in Togo.

$H$. combesi, a lung parasite of Conraua derooi is characterized by general body shape, ratio of sucker diameters $(2,7)$, slightly lobate ovary and testes, advanced development at the front of the uterine extra-cæcal loops, and extension of the vitelline glands into the posttesticular area. All these characters enable $H$. combesi to be distinguished from the other african species of the genus Haematoloechus, the closest of which is $H$. darcheni, a parasite of Conraua crassipes in Gabon.

En janvier 1973, nous avons récolté au Togo huit exemplaires de l'Amphibien Anoure Conraua derooi Hulselmans, 1971. Dans les poumons de deux d'entre eux, nous avons découvert un Trématode Digène dont nous donnons ci-dessous la description. 


\section{Haematoloechus combesi n. sp.}

Hôte: Conraua derooi Hulselmans, 1971.

Habitat : Poumons.

Localité : Klouto (Togo).

MATÉRIEL DE DESCRIPTION : 4 exemplaires.

\section{Description.}

\section{CORPS :}

Corps allongé, aplati dorso-ventralement (fig. 1). Le premier tiers de l'animal est rétréci; il est séparé du reste du corps par une sorte d'épaulement correspondant à la terminaison antérieure des boucles extracaecales de l'utérus. Cette disposition donne à l'espèce un aspect très caractéristique.

Les dimensions sont les suivantes:

- longueur : 5,2 à $6,1 \mathrm{~mm}$;

— largeur maximale : 2,8 à $3,3 \mathrm{~mm}$.

\section{TÉGUMENT :}

Tégument mince $(4,8 \mu)$, sans aucune trace de spinulation.

\section{Ventouses :}

Ventouse orale subterminale-ventrale, diamètre: 0,515 à $0,570 \mathrm{~mm}$.

Ventouse ventrale située un peu en avant de la mi-longueur du corps; diamètre : 0,170 à $0,230 \mathrm{~mm}$.

Rapport ventousaire VO/VV : 2,7 environ.

Distance entre centres des ventouses: $1,674 \mathrm{~mm}$.

Si la ventouse orale est fortement développée, l'acétabulum en revanche est très discret, quasi dépourvu de musculature, et ne paraît guère fonctionnel.

\section{APPAREIL DIGESTIF :}

Bouche donnant accès à un pharynx sub-sphérique mesurant 0,205 à $0,243 \mathrm{~mm}$ de diamètre ; rapport $\mathrm{VO} . \mathrm{Ph}$ : 2,5 environ. De part et d'autre de la région antérieure du pharynx existent deux petits amas glandulaires.

Esophage non discernable.

Caecums longs, se terminant près de l'extrémité postérieure du corps, contenant du sang en voie de digestion.

\section{APPAREIL REPRODUCTEUR MÂLE :}

Testicules intercaecaux, disposés en diagonale, post-acétabulaires. Leur forme épouse la place disponible entre les circonvolutions de l'utérus et apparaît comme légèrement lobée. Le testicule antérieur mesure environ 0,70 sur $0,58 \mathrm{~mm}$, le testicule postérieur 0,95 sur 


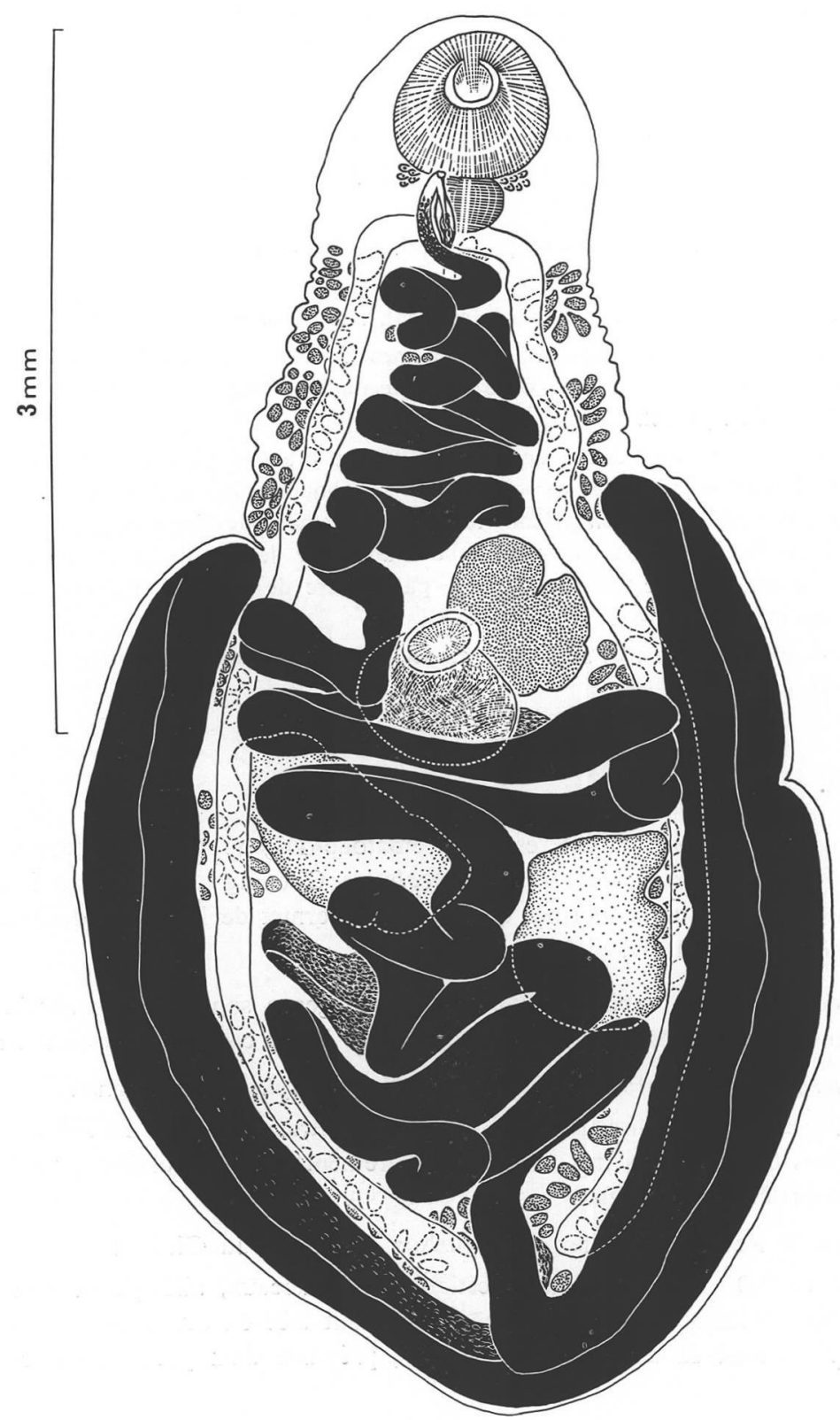

FIG. 1. - Haematoloechus combesi n.sp. - Vue ventrale. 
$0,48 \mathrm{~mm}$. Vésicule séminale masquée par l'utérus, aboutissant à une poche du cirre très réduite située au niveau du bord postérieur de la ventouse orale. La poche du cirre comprerd une vésicule séminale interne et des cellules prostatiques; elle débouche au pore génital commun.

\section{APPAREIL REPRODUCTEUR FEMELle :}

Ovaire intercaecal, para-acétabulaire, latéral, irrégulièrement mais profondément lołé. Il mesure environ 0,56 sur $0,60 \mathrm{~mm}$.

Réceptacle séminal immédiatement post-acétabulaire, subsphérique, presque aussi grand que l'ovaire.

Glande de Mehlis dorsale au réceptacle séminal, très développée.

Vitellogènes organisés en rosettes, elles-mêmes formées de follicules bien distincts. L'extension des vitellogènes recouvre celle des caecums digestifs; leur disposition précise est représentée sur la figure 2 .

Utérus comprenant une branche descendante et une branche ascendante formant toutes deux des circonvolutions puissantes et nombreuses. Les boucles utérines extracaecales, caractéristiques du genre Haematoloechus, sont ici particulièrement développées; elles remontent en avant de l'acétabulum jusqu'au niveau du bord antérieur de l'ovaire et sont responsables, comme nous l'avons souligné, de la silhouette particulière de l'animal. L'utérus aboutit au pore génital commun, au niveau du pharynx.

Oeufs brun clair à brun très sombre, à clapet visible, extrêmement nombreux, mesurant 0,022 sur $0,017 \mathrm{~mm}$.

\section{Discussion.}

Par l'ensemble de ses caractères, le Trématode Digène que nous avons décrit prend place dans la famille des Haematoloechidae Odening, 1964. La présence de deux ventouses et de boucles utérines extracaecales permet de le ranger dans le genre Haematoloechus Looss, 1899.

Actuellement, quatre espèces appartenant à ce genre sont connues sur le continent africain (1); nous les indiquons ci-après avec leurs caractères essentiels:

H. exoterorchis Rees, 1964, chez Dicroglossus occipitalis au Ghana:

$\mathrm{VO} / \mathrm{VV}=0,75$; ovaire régulier, testicules extracaecaux et fortement lobés ; boucles utérines extracaecales très réduites (entièrement post-testiculaires) ; vitellogènes s'étendant en arrière des testicules.

H. micrurus Rees, 1964, chez Dicroglossus occipitalis au Ghana:

$\mathrm{VO} / \mathrm{VV}=2,0$; ovaire régulier ; testicules intercaecaux, allongés et non lobés ; boucles utérines extracaecales très longues remontant très en avant de l'acétabulum ; vitellogènes s'étendant en arrière des testicules; présence d'un petit appendice postérieur.

(1) La présence en Afrique de Haematoloechus variegatus (Rudolphi, 1819), Looss, 1899, ne nous paraît pas devoir être considérée comme certaine. 


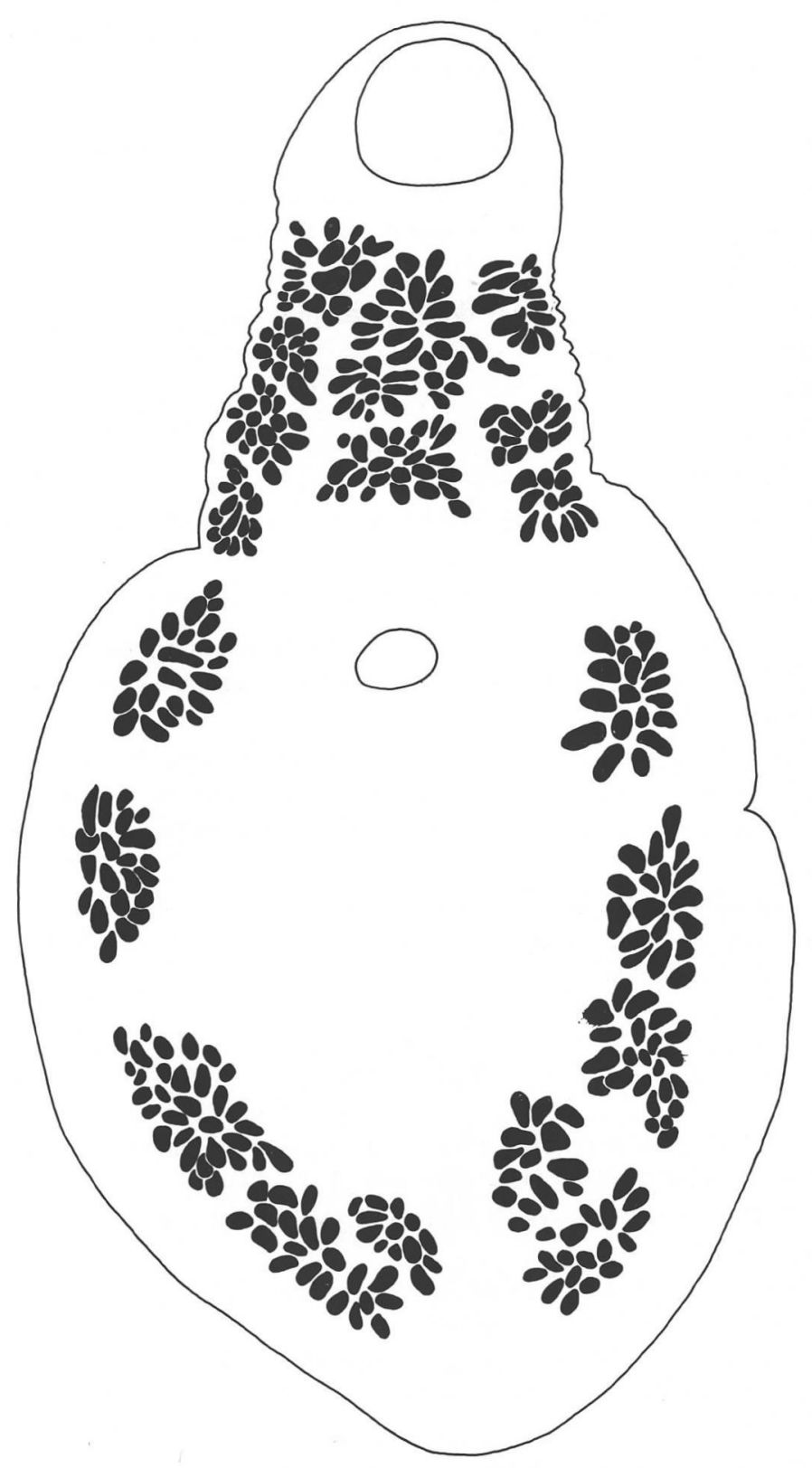

FIg. 2. - Haematoloechus combesi n. sp. - Disposition des glandes vitellogènes. 
H. darcheni Combes et Knoepffler, 1967, chez Conraua crassipes au Gabon:

$\mathrm{VO} / \mathrm{VV}=4,0$; ovaire très fortement lobé ; testicules intercaecaux et légèrement lobés; boucles utérines extracaecales atteignant le niveau des testicules; vitellogènes s'étendant en arrière des testicules.

H. lobogonadus Meskal, 1970, chez Rana angolensis en Ethiopie :

$\mathrm{VO} / \mathrm{VV}=2$; ovaire lobé ; testicules intercaecaux et fortement lobés ; boucles utérines extracaecales atteignant le niveau des testicules; vitellogènes ne s'étendant pas en arrière des testicules.

Nos exemplaires se distinguent des espèces précédentes par l'ensemble des caractères suivants :

$\mathrm{VO} / \mathrm{VV}=2,7$;

Ovaire lobé ;

Testicules intercaecaux et faiblement lobés;

Boucles utérines extracaecales dépassant antérieurement le niveau de l'acétabulum ;

Vitellogènes s'étendant en arrière des testicules.

On peut ajouter que la forme de nos exemplaires, marquée par un épaulement au niveau du rebroussement des boucles utérines extracaecales, n'existe pas chez les autres espèces.

Les affinités les plus étroites paraissent s'observer avec $H$. darcheni, également parasite d'un Conraua, mais $H$. darcheni possède un rapport ventousaire plus élevé, un ovaire beaucoup plus lobé et des boucles utérines extracaecales presque moitié moins longues.

Nous considérons le Trématode de Conraua derooi comme une espèce non décrite à ce jour que nous dédions à notre collègue Claude Combes, $\mathrm{P}^{\mathrm{r}}$ au Centre Universitaire de Perpignan.

\section{Bibliographie}

Combes (C.) et Knoepffler (L.-Ph.), 1967. - Parasites d'Amphibiens du Gabon Haematoloechidae (Digenea). Biologia Gabonica, 3 (2), 141-147.

Meskal (F.), 1970. - Trematodes of Anurans from Ethiopia. Acta universitatis Bergensis (1), 53-58.

Odening (K.), 1958. - Zur Systematic von Haematoloechus (Trematoda; Plagiorchiidae). Mitt. Zool. Mus. Berl., 34, 63-108.

-, 1960. - Revision der Unterfamilie Haematoloechidae Freitas and Lent, 1939 (Trematoda; Plagiorchiidae). Sond. Monats. Arad. Wiss, Berl., 2 (7), 449-454.

REEs (G.), 1964. - Two new species of the genus Haematoloechus (Digenea; Plagiorchiidae) from Rana occipitalis (Gunther) in Ghana. Parasitology, 54, 345-368. 